\title{
Erratum
}

\section{Gold(I)-Catalyzed Synthesis of Dihydrobenzofurans from Aryl Allyl Ethers}

Nicholas W. Reich, Cai-Guang Yang, Zhangjie Shi, Chuan He* Synlett 2006, 1278.

The advanced online publication (e-First) version of this article was missing the $\mathrm{R}^{1}$ group in the product structure of Table 2, entries 1 and 2. This error has been corrected both in the print version, which appears in this issue, and the current online version. 